\title{
The complex iron line of NGC 5506
}

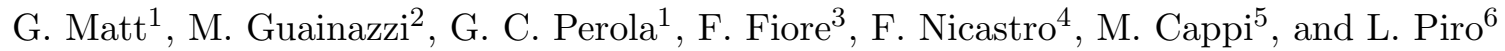 \\ 1 Dipartimento di Fisica, Università degli Studi Roma Tre, Via della Vasca Navale 84, 00146 Roma, Italy \\ 2 XMM-Newton SOC, VILSPA-ESA, Apartado 50727, 28080 Madrid, Spain \\ 3 Osservatorio Astronomico di Roma, Via dell'Osservatorio, 00044 Monteporzio Catone, Italy \\ 4 Harvard-Smithsonian Center of Astrophysics, 60 Garden Street, Cambridge MA 02138, USA \\ 5 Istituto Tecnologie e Studio Radiazioni Extraterrestri, CNR, Via Gobetti 101, 40129 Bologna, Italy \\ ${ }^{6}$ Istituto di Astrofisica Spaziale, C.N.R., Via Fosso del Cavaliere, 00133 Roma, Italy \\ Received 31 July 2001 / Accepted 29 August 2001
}

\begin{abstract}
The bright Narrow Emission Line Galaxy, NGC 5506, has been observed simultaneously by XMMNewton and BeppoSAX. The iron line is complex, with at least two components: one narrow and corresponding to neutral iron, the second one broad and corresponding to ionized iron. The latter line is equally well fitted by a truly broad line or by a blend of $\mathrm{He}^{-}$- and $\mathrm{H}$-like narrow lines. The bulk of the Compton reflection continuum originates in neutral matter, and is therefore associated with the narrow line: they are most likely emitted in distant matter. The origin of the ionized line(s) is less certain, but the solution in terms of a blend of narrow lines from photoionized matter seems to be preferable to emission from an ionized, relativistic accretion disc.
\end{abstract}

Key words. galaxies: individual: NGC 5506 - galaxies: Seyfert - X-rays: galaxies

\section{Introduction}

Recent Chandra (e.g. Kaspi et al. 2001; Yaqoob et al. 2001) and XMM-Newton (e.g. Reeves et al. 2001; Pounds et al. 2001; Gondoin et al. 2001) results clearly indicate that a narrow iron line component is often, if not always, present in Seyfert 1 galaxies, alone or together with the relativistic disc component (Fabian et al. 2000 and references therein). An analysis of the composite $A S C A$ spectrum of Seyfert 1s (Lubinski \& Zdziarski 2001) also suggests the presence of a narrow component, confirming previous findings on individual sources. Whether this component arises in Compton-thin matter like the Broad Line Region or the Narrow Line Region, or in Comptonthick material like the "torus" (Antonucci 1993) or the outflowing matter (Elvis 2000) envisaged in Unification Models and clearly present in several Seyfert 2s (Maiolino et al. 1998; Risaliti et al. 1999; Matt 2001 and references therein), is however still a matter of debate. The high energy resolution Chandra observation of NGC 5548 (Yaqoob et al. 2001) just resolved the line width, which comes out to be consistent with those of the optical broad lines. The upper limit on the line width in NGC 3783 (Kaspi et al. 2001) is instead consistent with the iron line originating outside the BLR.

In this paper we present the results from a XMMNewton observation of NGC 5506 simultaneous with a BeppoSAX observation, aimed to study in unprecedented

Send offprint requests to: G. Matt, e-mail: matt@fis.uniroma3.it detail the reprocessed components by combining the sensitivity of XMM-Newton at the iron line energy with the still unique capability of BeppoSAX in hard X-rays.

The Narrow Line Emission Galaxy NGC 5506 is one of the brightest AGN in hard X-rays, and for this reason has been extensively studied in the past. The nucleus is obscured by cold matter with a column density of about $3 \times 10^{22} \mathrm{~cm}^{-2}$. A possibly variable soft component (Bond et al. 1993) was discovered by GINGA, which also detected the iron $\mathrm{K} \alpha$ line and the reflection component. Both $A S C A$ spectroscopic (Wang et al. 1999) and $R X T E$ variability (Lamer et al. 2000) observations suggest that the line is complex. BeppoSAX (Perola et al. 2001) found an iron line centroid energy bluer than $6.4 \mathrm{keV}$, i.e. $E_{k}=$ $6.52 \pm 0.09$ (equivalent width of $150 \pm 40 \mathrm{eV}$ ) and measured the reflection component at $R=1.2 \pm 0.4$. Only a lower limit of $300 \mathrm{keV}$ could be put on the high-energy exponential cut-off.

\section{Observations and data analysis}

XMM-Newton (Jansen et al. 2001) observed NGC 5506 between February 2 (17:34 UT) and February 32001 (03:35 UT). Imaging CCD cameras (EPIC-MOS, 0.2$10 \mathrm{keV}$, Turner et al. 2001; EPIC-p-n, 0.2-15 keV, Strüder et al. 2001) were operated in Large Window mode, with the Medium filter. High resolution spectroscopy cameras (RGS; $0.2-1.5 \mathrm{keV}$; der Herder et al. 2001) were simultaneously operating, but - due to the large absorbing column - they detected the source only at the $\simeq 4 \sigma$ level. No evidence for narrow absorption or emission structure 
is present in the RGS spectra, and we will not deal with these data any longer in this paper. Data were reduced with SAS V.5.0 (Jansen et al. 2001), using the calibration files publicly available on April 2001. After data screening the total exposure time was $17.6 \mathrm{ks}$ and $13.8 \mathrm{ks}$ for the MOS and the $\mathrm{p}-\mathrm{n}$, respectively. X-ray events corresponding to pattern $0-12$ for the MOS and to pattern 0 for the $\mathrm{p}-\mathrm{n}$ were used. Non X-ray background remained low throughout the observation. Spectra and light curves were extracted in regions of $40^{\prime \prime}$ radii. The $\mathrm{p}-\mathrm{n}$ count rate in the $0.1-15 \mathrm{keV}$ band is $3.75 \pm 0.16$, corresponding to a pile-up fraction of $\simeq 0.5 \%$ only (in the MOS the pile-up fraction is about $0.7 \%$ ). In particular, the $\mathrm{p}-\mathrm{n}$ count rate in the 6-7 keV range is 3 times than in ASCA/SIS, making it the best CCD instrument ever for iron line studies.

BeppoSAX observed the source from February 1 (5:50 UT) to February 3 (13:20 UT), 2001, for a total net exposure time of $78 \mathrm{ks}$ for the MECS and PDS (2 units) instruments, and $29 \mathrm{ks}$ for the LECS. Data reduction and analysis were standard (e.g. Guainazzi et al. 1999). LECS and MECS spectra and light curves were extracted in regions of $4^{\prime}$ radii.

The source varied in flux by about $40 \%$ during the BeppoSAX observation, and by about $20 \%$ during the $\mathrm{XMM}-N$ ewton observation. No spectral variability has been detected.

In this paper we used only the XMM/EPICp-n and BeppoSAX/PDS instruments after having checked that the spectra from XMM/EPIC-MOS and BeppoSAX/MECS instruments were consistent with that of XMM/EPIC-p-n. (A temporal and spectral analysis of the complete XMM-Newton and BeppoSAX data sets is beyond the scope of this paper and is to a future work.) In order to maximize the statistics, and because of the lack of spectral variability, we integrated the PDS spectrum over the entire observation despite only a part of it was covered by the XMM-Newton observation. In fitting the $\mathrm{p}-\mathrm{n}$ and PDS spectra we introduced a multiplicative factor of 1.215 for the PDS to take into account crosscalibrations and the different exposure times. This factor was obtained by normalizing the p-n flux to the integrated MECS spectrum and applying a relative normalization factor of 0.84 between the BeppoSAX PDS and MECS (Fiore et al. 1999).

Spectral analysis has been performed with the XSPEC V.11 software package. All errors refer to $90 \%$ confidence level for 1 interesting parameter $\left(\Delta \chi^{2}=2.7\right)$.

\section{Results}

\subsection{The baseline model}

In Fig. 1 the p-n/PDS spectrum is shown, together with the residuals after fitting the data with a simple absorbed power-law model. A soft excess and a strong iron line are clearly apparent in the residuals. The high energy part of the spectrum is also badly fitted. A blow-up of the residuals around the iron line shows clearly that the line is

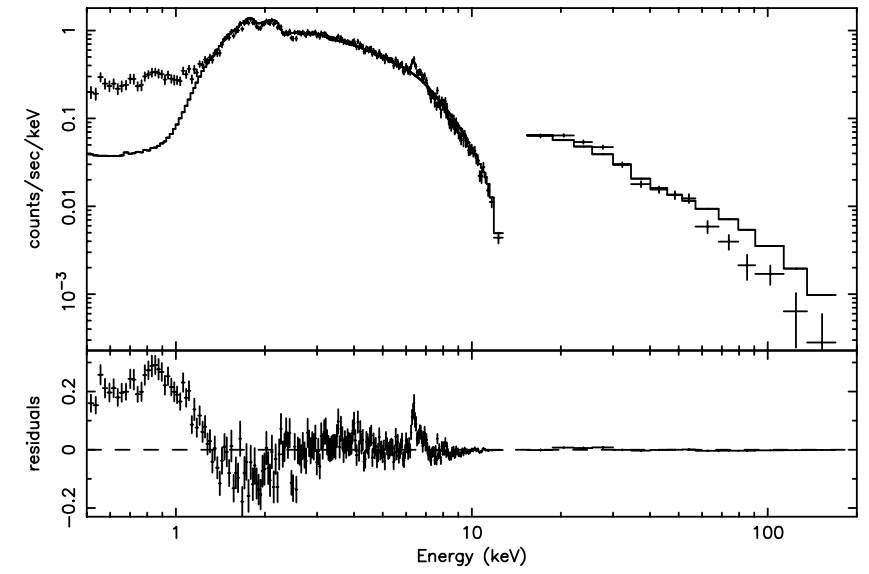

Fig. 1. The EPIC-p-n and BeppoSAX-PDS spectra fitted with a simple absorbed power law.

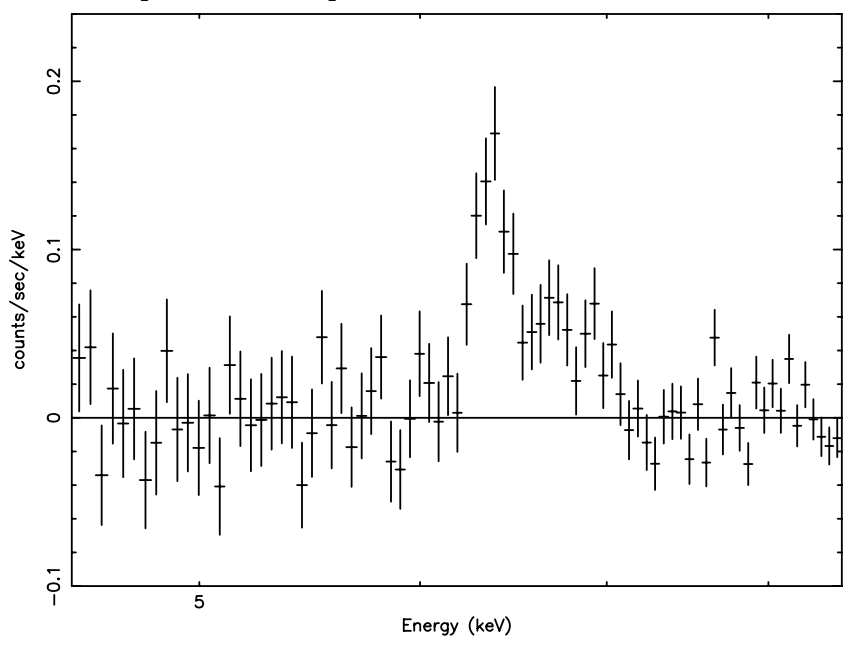

Fig. 2. Residuals in the iron line region. At least two iron line components are clearly present.

complex, being composed by a narrow component around $6.4 \mathrm{keV}$ and a broader component bluewards.

Because in this paper we are mainly interested in the reprocessed components, for the sake of simplicity we excluded from the analysis the energies below $2.5 \mathrm{keV}$, where the soft excess is present.

We first added to the model two gaussian lines. One of the lines turns out to be unresolved and close to $6.4 \mathrm{keV}$, the second one broad and corresponding to ionized iron. The quality of the fit improves, but it is still unacceptable $\left(\chi_{\mathrm{r}}^{2} /\right.$ d.o.f. $\left.=243.9 / 173\right)$. Because most of the $\chi^{2}$ comes from the highest energy part of the spectrum, we added also a reflection component (PEXRAV model: Magdziarz \& Zdziarski 1995). The fit is now perfectly acceptable: $\chi_{\mathrm{r}}^{2} /$ d.o.f. $=176.4 / 172$. (We note here that a fit with only one gaussian line, which basically fits the narrow component, gives $\chi_{\mathrm{r}}^{2} /$ d.o.f. $=202.9 / 175$. The ionized line is therefore significant at more than $99.99 \%$ confidence level, according to the F-test.) The best fit parameters are summarized in Table 1.

The fit without the PDS gives similar values for the iron lines and the power law index, but only an upper limit to $R$ of 1.6 . 
Table 1. Baseline Model Spectrum fit ( $i$ is fixed to $30^{\circ}$ in the $\mathrm{CR}$ model). Line energies are in the source rest frame $(z=0.0061)$.

\begin{tabular}{|c|c|}
\hline$F(2-10 \mathrm{keV})\left(\mathrm{erg} \mathrm{cm}^{-2} \mathrm{~s}^{-1}\right)$ & $7.8 \times 10^{-11}$ \\
$\Gamma$ & $1.98_{-0.02}^{+0.05}$ \\
$N_{\mathrm{H}}\left(10^{22} \mathrm{~cm}^{-2}\right)$ & $3.44_{-0.12}^{+0.13}$ \\
$E_{\mathrm{f}}(\mathrm{keV})$ & $330_{-80}^{+120}$ \\
$R$ & $1.09_{-0.08}^{+0.08}$ \\
$E_{k, 1}(\mathrm{keV})$ & $6.41_{-0.03}^{+0.03}$ \\
$\sigma_{k, 1}(\mathrm{keV})$ & $<0.06$ \\
$I_{k, 1}\left(\mathrm{ph} \mathrm{cm} \mathrm{s}^{-1}\right)$ & $5.0_{-0.7}^{+2.2} \times 10^{-5}$ \\
$E W_{k, 1}(\mathrm{eV})$ & $70_{-10}^{+30}$ \\
$E_{k, 2}(\mathrm{keV})$ & $6.75_{-0.15}^{+0.10}$ \\
$\sigma_{k, 2}(\mathrm{keV})$ & $0.25_{-0.06}^{+0.08}$ \\
$I_{k, 2}\left(\mathrm{ph} \mathrm{cm} \mathrm{s}^{-2}\right)$ & $7.0_{-1.9}^{+1.8} \times 10^{-5}$ \\
$E W_{k, 2}(\mathrm{eV})$ & $110 \pm 30$ \\
$\chi^{2} /$ d.o.f. & $176.4 / 172$ \\
\hline
\end{tabular}

To be sure that the above findings do not depend on the limited energy band adopted, we repeated the above analysis extending the energy band down to $0.5 \mathrm{keV}$. The soft excess is well fitted by a partial covering model with covering fraction of about $98 \%$, plus a further, complete absorber with column density of about $2 \times 10^{21} \mathrm{~cm}^{2}$. Again, the inclusion of a second Gaussian line is highly significant $\left(\chi_{\mathrm{r}}^{2} /\right.$ d.o.f. $=318.9 / 250$ for one line, and $289.5 / 247$ for two lines; the second line is significant at more than 99.99\% confidence level). The best-fit parameters are all consistent within the errors with those reported in Table 1.

\subsection{The origin of the iron lines}

The $6.4 \mathrm{keV}$ iron line is clearly too narrow to come from the innermost part of the accretion disc. It may arise either in Compton-thin material, like the Broad Line Region or Narrow Line Region, or in Compton-thick matter, like the outermost part of the accretion disc or the "torus". To distinguish between the two possibilities it is necessary to understand whether the Compton Reflection (CR) component is at least partly associated with the $6.4 \mathrm{keV}$ line (so indicating emission from Compton-thick matter) or, instead, is completely associated with the ionized line (in which case the matter responsible for the narrow line must be Compton-thin).

We first tested whether the CR may be completely associated with the ionized line, supposing that both are emitted in a relativistic disc. We accounted for relativistic effects in both the line and CR by adopting the models DISKLINE (Fabian et al. 1989) and REFSCH, respectively. All disc parameters were forced to be the same in the DISKLINE and REFSCH models. We fixed the inner radius to $6 r_{\mathrm{g}}$ (the innermost stable orbit in Schwarzschild metric) and left the outer radius and the inclination angle as free parameters. (For simplicity, here and in all following fits, the energy of the unresolved line has been fixed to $6.4 \mathrm{keV}$, and its width to zero. ) The fit is good $\left(\chi^{2} /\right.$ d.o.f. $=$ 174.6/172) but the results not physically self-consistent: in fact, while the line rest frame energy confirms that the iron must be ionized $\left(E_{\mathrm{k}}=6.78 \pm 0.08 \mathrm{keV}\right)$, the ionization parameter of the reflecting matter is very low $(\xi<$ $0.17 \mathrm{erg} \mathrm{cm} \mathrm{s}^{-1}$ ) and definitely inconsistent with the ionization inferred from the iron line.

After fixing, for simplicity, the energy of the iron line to $6.7 \mathrm{keV}$, corresponding to He-like iron, we forced the disc to be really ionized, i.e. with the ionization param-

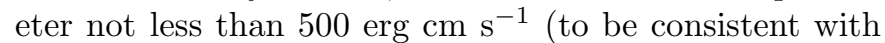
the centroid energy of the ionized iron line). The fit is totally unacceptable $\left(\chi^{2} /\right.$ d.o.f. $=339.9 / 173$; see Fig. 3$)$, due to a bad fitting of the continuum at low energies and to a deep $(\tau \sim 0.2)$ edge at $7.1 \mathrm{keV}$, which in the previous fits were accounted for by the neutral reflection continuum. We checked whether this edge may be related to the cold absorber by allowing the iron abundance to vary with respect to the other elements. A much better fit $\left(\chi^{2} /\right.$ d.o.f. $\left.=216.0 / 172\right)$ is found with $A_{\mathrm{Fe}} \sim 10$, but still significantly worse than for the baseline model discussed above. Moreover, the iron overabundance seems unrealistically large. Therefore, we conclude that most if not all of the CR comes from neutral matter and must therefore be associated with the cold, narrow iron line. The ratio between the neutral iron line equivalenth width and the amount of Compton reflection component is somewhat lower than expected (an $E W$ of about $130 \mathrm{eV}$ is predicted if $R=1, \theta=30^{\circ}$ and $\Gamma=2$, George \& Fabian 1991; Matt et al. 1991), suggesting a possible iron underabundance.

The nature of the ionized line is less clear. The disc solution is possible but rather unlikely. Including in the model both a cold and an ionized CR, a good fit $\left(\chi^{2} /\right.$ d.o.f. $\left.=174.7 / 172\right)$ is found, but with the cold reflection largely dominating $\left(R_{\text {cold }}=1.35_{-0.25}^{+0.50}, R_{\text {ion }}<0.28\right)$, implying a rather large ratio between the ionized iron line $E W(\sim 100 \mathrm{eV})$ and the corresponding CR. Such a ratio is indeed possible when iron is mainly in the He-like stage (Matt et al. 1993, 1996; Ballantyne et al. 2001; Nayakshin \& Kallman 2001); the small value of the CR could be due to either an almost edge--on disc or a small emitting region. The inclination angle of the disc is not well constrained in this fit, any value above $25^{\circ}$ being possible. However, because the ionized line is not very broad, the best fit value for the outer radius is pretty large (lower limit of about $300 R_{\mathrm{g}}$ ), while any realistic disc model suggests strong ionization only in the innermost regions.

Alternatively, the line may be a blend of two or more narrow lines. A fit with a blend of $\mathrm{He}$-like and $\mathrm{H}$-like lines is as good as that with the broad line $\left(\chi^{2} /\right.$ d.o.f. $=$ $172.9 / 175)$. The $E W$ of the two lines are $40 \pm 16 \mathrm{eV}$ and $32 \pm 15 \mathrm{eV}$, respectively. (The $E W$ of the neutral line is now $90 \pm 15 \mathrm{eV}$, other parameters similar to those in Table 1.) These ionized lines can be produced by fluorescence and resonant scattering in photoionized matter (Matt et al. 1996). Equivalent widths similar to the 


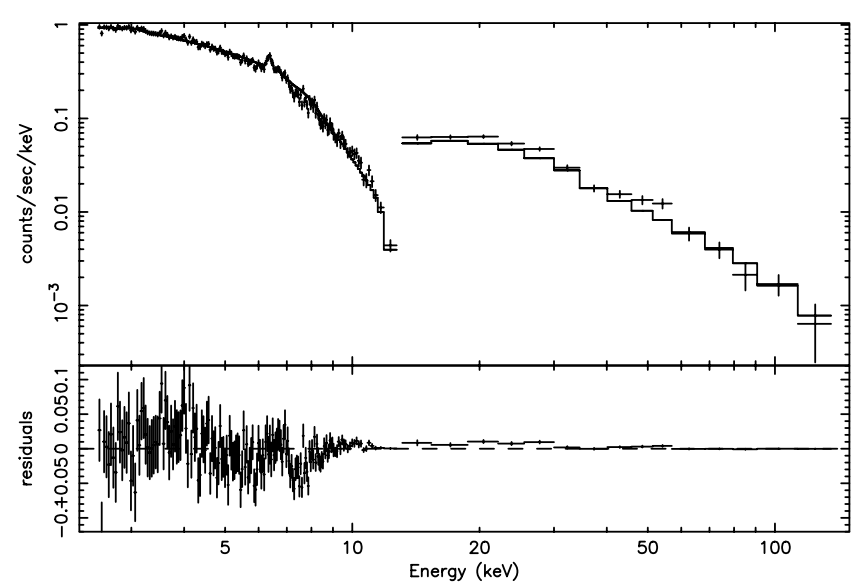

Fig. 3. Spectra and best-fit model with only the ionized reflection component. See text for details.

observed ones may be obtained if the Thomson optical depth of the ionized matter is a few hundredths (Bianchi et al., in preparation). Interestingly, the normalization of the soft excess is about $2 \%$ of the primary component. It is then possible that the soft $\mathrm{X}$-ray emission and the ionized lines come from one and the same matter.

\section{Discussion and conclusions}

The iron line in NGC 5506 is clearly complex. The cold and narrow component is associated with the bulk of the Compton Reflection emission and therefore originates in Compton-thick matter, like the torus. In NGC 5506 absorption is instead Compton-thin. This implies either that the torus is dishomogeneous, or that the reflector and the absorber are different altogether (e.g. Matt 2000). This is one of the few cases in which distant, Compton-thick matter is observed in reflection only. (The best case so far was NGC 4051, Guainazzi et al. 1998.) If this component will be found to be common in type 1 Seyfert, this will support one of the least tested predictions of unification models, i.e. that any Seyfert 1 is surrounded by circumnuclear, optically thick matter.

The origin of the ionized component is less clear, but a solution in terms of a blend of narrow $\mathrm{He}-$ and $\mathrm{H}$-like iron lines seems preferable to that of a relativistic, ionized disc. If this is indeed the case, we are left with no evidence whatsoever for disc emission, a rather puzzling situation. Of course, a trivial possibility is that the disc is nearly edge- on, reducing the reprocessed components to invisibility. This solution can certainly work for a single source, but would be untenable if the same situation would occur in many other sources.

The iron line complex observed in NGC 5506 is very similar to those observed by XMM-Newton in Mrk 205 (Reeves et al. 2001) and Mrk 509 (Pounds et al. 2001). One cannot help wondering if this is not the rule for Seyfert galaxies. However, in the XMM-Newton observation of Fairall 9 (Gondoin et al. 2001) only the narrow and cold component is detected. Moreover, NGC 5506 and Mrk 509 are the only two sources in the BeppoSAX sample of Perola et al. (2001) in which the iron line centroid energy is higher than $6.4 \mathrm{keV}$. It is therefore possible that these sources represent the exception rather than the rule.

Acknowledgements. We thank the BeppoSAX Scientific Data Center and the User Support Group of the XMM-Newton SOC for their help. G. M., G. C. P. and F. F. acknowledge financial support from ASI and from MURST (under grant COFIN-00-02-36).

\section{References}

Antonucci, R. 1993, ARA\&A, 31, 473

Ballantyne, D. R., Iwasawa, K., \& Fabian, A. C. 2001, MNRAS, in press

Bond, I. A., Matsuoka, M., \& Yamauchi, M. 1993, ApJ, 405, 179

der Herder, J. W., Brinkman, A. C., Kahn, S. M., et al. 2001, A\&A, 365, L7

Elvis, M. 2000, ApJ, 545, 63

Fabian, A. C., Rees, M. J., Stella, L., \& White, N. E. 1989, MNRAS, 238, 729

Fabian, A. C., Iwasawa, K., Reynolds, C. S., \& Young, A. J. 2000, PASP, 112, 1145

Fiore, F., Guainazzi, M., \& Grandi, P. 1999, SDC report (available from http://www.asdc.asi.it/bepposax/ software/index.html)

George, I. M., \& Fabian, A. C. 1991, MNRAS, 249, 352

Gondoin, P., Lumb, D., Siddiqui, H., Guainazzi, M., \& Schartel, N. 2001, A\&A, 373, 805

Guainazzi, M., Nicastro, F., Fiore, F., et al. 1998, MNRAS, 301, L1

Guainazzi, M., Perola, G. C., Matt, G., et al. 1999, A\&A, 346, 407

Kaspi, S., Brandt, W. N., Netzer, H., et al. 2001, ApJ, 554, 216

Jansen, F., Lumb, D., Altieri, B., et al. 2001, A\&A, 365, L1

Lamer, G., Uttley, P., \& McHardy, I. M. 2000, MNRAS, 319, 949

Lubinski, P., \& Zdziarski, A. A. 2001, MNRAS, 323, L37

Magdziarz, P., \& Zdziarski, A. A. 1995, MNRAS, 273, 837

Maiolino, R., Salvati, M., Bassani, L., et al. 1998, A\&A, 338, 781

Matt, G., Perola, G. C., \& Piro, L. 1991, A\&A, 247, 25

Matt, G., Fabian, A. C., \& Ross, R. R. 1993, MNRAS, 262, 179

Matt, G., Fabian, A. C., \& Ross, R. R. 1996, MNRAS, 278, 1111

Matt, G., Brandt, W. N, \& Fabian, A. C. 1996, MNRAS, 280, 823

Matt, G. 2000, A\&A, 355, L13

Matt, G. 2001, in Issues in Unification of AGNs, ed. R. Maiolino, A. Marconi, \& N. Nagar, ASP Conf. Ser., in press [astro-ph/0107584]

Nayakshin, S., \& Kallman, T. R. 2001, ApJ, 546, 406

Perola, G. C., et al. 2001, in preparation

Pounds, K. A., Reeves J. N., O'Brien P. T, et al. 2001, ApJ, in press

Reeves, J. N., Turner, M. J. L., Pounds, K. A., et al. 2001, A\&A, 365, L134

Risaliti, G., Maiolino, R., \& Salvati, M. 1999, ApJ, 522, 157

Strüder, L., Briel, U., Dennerl, K., et al. 2001, A\&A, 365, L18

Turner, M. J. L., Abbey, A., Arnaud, M., et al. 2001, A\&A, $365, \mathrm{~L} 27$

Wang, T., Mihara, T., Otani, C., Matsuoka, M., \& Awaki, H. 1999, ApJ, 515, 567

Yaqoob, T., George, I. M., Nandra, K., et al. 2001, ApJ, 546, 759 